\title{
The diagnosis and the reproductive and metabolic consequences of polycystic ovary syndrome
}

\author{
Mohannad AbuFaza', Ibrahim A. Abdelazim², Prashant Purohit' ${ }^{3}$, Svetlana Shikanova ${ }^{4}$, \\ Gulmira Zhurabekova ${ }^{5}$, Bakyt Karimova ${ }^{4}$, Mukhit Sarsembayev ${ }^{4}$, Tatyana Starchenko ${ }^{4}$ \\ 'Department of Obstetrics and Gynecology, Ahmadi Hospital, Ahmadi, Kuwait \\ 2Department of Obstetrics and Gynecology, Ahmadi Hospital, Kuwait and Ain Shams University, Cairo, Egypt \\ ${ }^{3}$ Department of Reproductive Medicine and Assisted Conception, Kings College Hospital, London, UK \\ ${ }^{4}$ Department of Obstetrics and Gynecology No. 1, Marat Ospanov, West Kazakhstan State Medical University (WKSMU), Aktobe, \\ Kazakhstan \\ ${ }^{5}$ Department of Normal and Topographical Anatomy, Marat Ospanov, West Kazakhstan State Medical University (WKSMU), \\ Aktobe, Kazakhstan
}

\begin{abstract}
Polycystic ovary syndrome (PCOS) is the most common endocrine disorder affecting women in their reproductive age, with significant reproductive, metabolic and psychological implications. The PCOS is a multifactorial disorder involving interactions between certain genes, environmental factors, gonadotropin dysfunction, insulin resistance (IR) and hyperandrogenism. Different phenotypes of PCOS exist, based on the PCOS definition by NIH and ESHRE/ARM and the clinical presentation. A large proportion of PCOS women have high luteinizing hormone (LH) levels. Partly due to the increased LH stimulation there is increased ovarian androgen production. The high androgen levels were suggested as the main cause of ovarian follicle maturation arrest with subsequent infertility. In addition, insulin stimulates and maintains ovarian androgen secretion, which explains the association between hyperinsulinemia and the severity of hirsutism in PCOS. There is no ideal therapy that treats all features of PCOS, so the treatment is directed towards the clinical presentation (targeted therapy).

Key words: diagnosis, reproductive, metabolic, polycystic ovary syndrome.
\end{abstract}

Corresponding author: Ibrahim A. Abdelazim, Department of Obstetrics and Gynecology, Ain Shams University, Egypt and Consultant at Ahmadi Hospital, Kuwait Oil Company (KOC), P.O. Box: 9758, 61008 Ahmadi, Kuwait, phone: +965 66551300, e-mail: dr.ibrahimanwar@gmail.com 


\section{Introduction}

Polycystic ovary syndrome (PCOS) is the most common endocrine disorder affecting women in their reproductive age, with significant reproductive, metabolic and psychological implications [1].

Reproductive implications include chronic anovulation, infertility, hyperandrogenism and hirsutism. Metabolic implications include insulin resistance (IR), impaired glucose tolerance (IGT), type 2 diabetes mellitus (DM2) and adverse cardiovascular disorders (CVDs). Psychological implications include anxiety and depression [2].

The prevalence of PCOS is about 5-6\%, but the prevalence rates reported are naturally dependent on the definition used and on the ethnicity of the studied population [3].

The prevalence of PCOS varies depending on which criteria are used to make the diagnosis, but is as high as 15$20 \%$ when the ESHRE/ASRM criteria are used for the diagnosis of PCOS. In addition, Ibrahim et al. reported 5-6\% prevalence of PCOS in Kuwaiti women and Schmidt et al. reported 5-10\% prevalence of PCOS in Sweden women $[1,3]$. The PCOS is multifactorial and multiple causative mechanisms have been suggested, involving interactions between certain genes, environmental factors, gonadotropins dysfunction, IR, hyperinsulinemia and hyperandrogenism. Because PCOS women usually have a family history of PCOS, researchers have suggested that a genetic variation at the androgen receptor and not just loss-of-function mutations is the main cause of hyperandrogenism in PCOS [4, 5]. In addition, they suggested that gene variation in PCOS occurs at the aromatase gene (SNP rs2414096 of the CYP19 gene) with subsequent aromatase deficiency or reduced aromatase activity and they found that the antral follicles taken from PCOS populations exhibited no aromatase activity [611]. Aromatase deficiency or reduced aromatase activity in the ovarian follicles with subsequent androgen excess (Figure 1) might contribute to abnormal follicle development seen in PCOS [12-15]. Prenatal exposure to androgens and weight gain have also been suggested as factors contributing to PCOS.

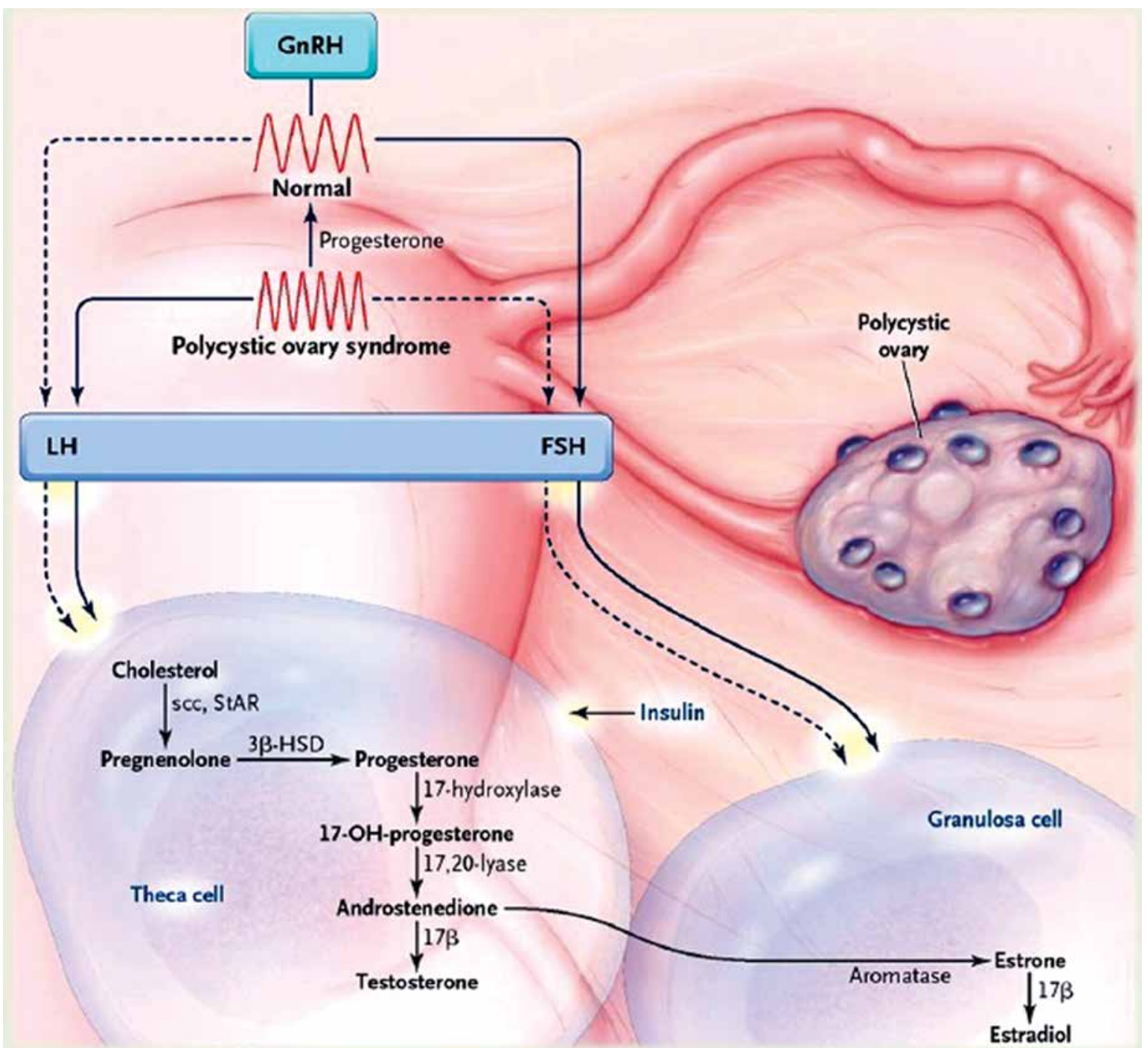

Figure 1. Two-cell theory (theca and granulosa cell theory) of ovarian steroidogenesis and the aromatase deficiency or reduced aromatase activity in PCOS women, which prevent conversion of androstenedione from the theca cell to estrogen (estrone $\mathrm{E}_{1}$ and estradiol $\mathrm{E}_{2}$ ) in the granulosa cell with subsequent increased ovarian androgen mainly androstenedione 
Different phenotypes of PCOS exist based on the PCOS definition by NIH and ESHRE/ARM and the clinic presentation of PCOS: either PCOS with oligo/amenorrhea alone or PCOS with oligo/amenorrhea and hyperandrogenism or PCOS with oligo/amenorrhea, hyperandrogenism and metabolic syndrome (MS).

\section{Diagnosis of polycystic ovary syndrome}

\section{$\mathrm{NIH/ESHRE/ARM} \mathrm{criteria}$}

NIH and ESHRE/ARM 2003 diagnostic criteria of PCOS are based on the clinical identifications of at least two of the three following criteria: 1) polycystic ovary (PCO) at ultrasound examination ( $\geq 10$ small follicles measuring $2-8 \mathrm{~mm}$ in both ovaries), 2) oligo or amenorrhea/anovulation, 3) clinical or biochemical hyperandrogenism after exclusion of excess androgen such as late onset adrenal hyperplasia, Cushing's syndrome and androgen-secreting tumors [4].

The diagnosis of PCOS is only established after exclusion of endocrine disorders causing irregular menses and excess androgen using the following facilities:

1) Pelvic ultrasound (multiple small follicles) $\geq 10$ small follicles measuring 2-8 $\mathrm{mm}$ in both ovaries.

2) Reproductive hormones: $\mathrm{LH} /$ follicle-stimulating hormone (FSH) ratio $2 / 1$ or $3 / 1$ (normally $1 / 1$ ), thyroid profile and prolactin.

3) Androgen profile (17-OH progesterone to exclude late onset adrenal hyperplasia, total testosterone to exclude androgen secreting tumors (when the total testosterone $>8.7 \mathrm{mmol} / \mathrm{l}(250 \mathrm{ng} / \mathrm{dl})$ androgen secreting tumors suspected which confirmed by computed tomography (CT) and/or magnetic resonance imaging (MRI)).

4) 24-hour urinary cortisol and dexamethasone suppression test to exclude Cushing's syndrome.

5) Two-hour oral glucose tolerance test (OGTT) that measures fasting, postprandial glucose, insulin level, triglyceride and high-density lipoprotein (HDL) cholesterol for detection of IR and MS in PCOS women.

\section{Morphology of polycystic ovaries}

Stein and Leventhal described PCO macroscopically as bilaterally enlarged ovaries, globular in shape. The presence of multiple cysts, less than $15 \mathrm{~mm}$, lined by hypertrophic theca cells and thick tunica albuginea immediately below the ovarian surface epithelium was described as histological features of PCO by Stein and Leventhal $[1,3,4]$. Figure 2 shows a laparoscopic view of PCO as bilaterally enlarged ovaries, globular in shape.

After the introduction of ultrasound in gynecology, the ultrasound diagnostic criteria for PCO replaced the macroscopic and histological criteria of PCO. The presence of $\geq 12$ follicles measuring 2-9 $\mathrm{mm}$ in diameter, and/or at least one enlarged $\left(>10 \mathrm{~cm}^{3}\right)$ ovary, is defined as PCO by ultrasound $[3,4]$. Figure 3 shows ultrasound or sonographic appearance of PCO with $\geq 12$ follicles measuring $2-9 \mathrm{~mm}$ in diameter.

\section{Clinical features of polycystic ovary syndrome}

Polycystic ovary syndrome is characterized by oligo/ amenorrhea, clinical or biochemical hyperandrogenism and polycystic ovaries. Oligo/amenorrhea is an indicator of oligo/anovulation and associated with infertility. Oligomenorrhea is defined as a menstrual interval of more than 35 days and amenorrhea is defined as the absence of menstrual bleeding for more than 90 days. Hyperandrogenism is caused by increased ovarian and/or increased adrenal androgen production. The typical symptoms of hyperandrogenism are hirsutism, acne and/or androgen alopecia. The clinical hyperandrogenism of PCOS diagnosed by the presence of masculine hair pattern distribution and the Ferriman-Gallwey system is a scoring system widely used today to define the extent of hirsutism [3]. Biochemical hyperandrogenism of PCOS is diagnosed by the free androgen index (FAI) being above the $95^{\text {th }}$ percentile of the reference value applied in the laboratory used for the analysis for the androgen sample. The FAI is calculated as the ratio between total testosterone divided by sex hormone-binding globulin $($ SHBG $) \times 100$. Polycystic ovary syndrome is also associated with obesity, IR, hyperinsulinemia, DM2, hypertension and dyslipidemia (metabolic syndrome).

Depending on the criteria used, $66-75 \%$ of PCOS women present with menstrual dysfunction and 60-69\% present with hirsutism and/or acne [3]. Weight gain in PCOS women is associated with aggravation of the PCOS symptoms,

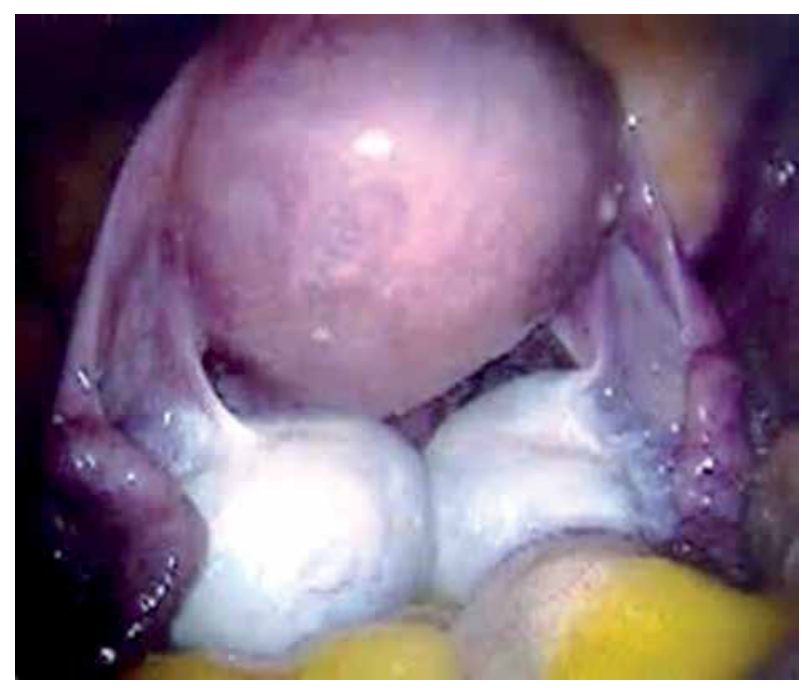

Figure 2. Laparoscopic view of PCO as bilaterally enlarged ovaries, globular in shape

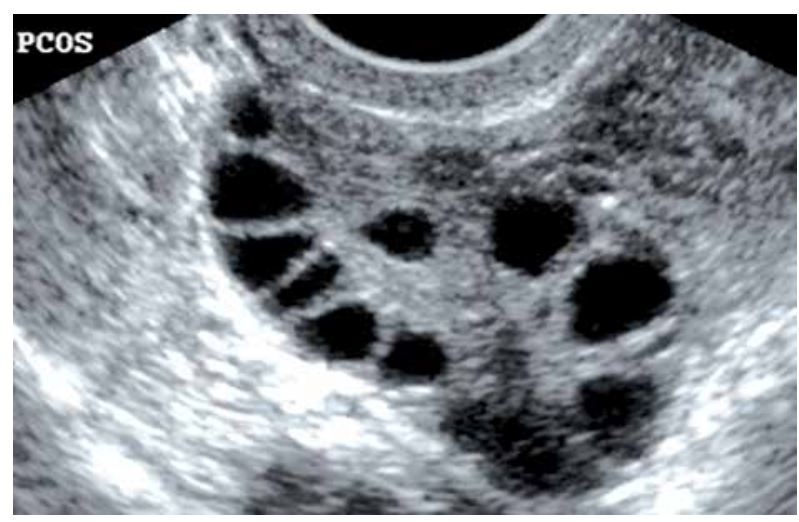

Figure 3. Sonographic appearance of $\mathrm{PCO} \geq 12$ follicles measuring 2-9 $\mathrm{mm}$ in diameter 
while weight loss of $5-10 \%$ is associated with significant improvement and regression of the reproductive features of the syndrome [3]. Five-ten percent effective weight loss in PCOS women can reverse the PCOS-associated anovulation with subsequent return of normal regular menstrual flow and fertility.

\section{Metabolic consequences of polycystic ovary syndrome}

\section{Obesity, weight gain}

Obesity is a chronic disease worldwide and obese PCOS women are exposed to increased risk of metabolic syndrome (IGT, DM2 and CVDs) [1, 3]. At least 35\% of women with PCOS are obese and the obesity of PCOS women is characteristic by body mass index $(\mathrm{BMI})>26 \mathrm{~kg} / \mathrm{m}^{2}$ with increase in the waist circumference $>35$ inches (waist circumference in centimeters measured midway between the upper border of the iliac crest and the lower rib margin) [1]. Generally, $10-40 \%$ of PCOS women are known to be obese (BMI $>30 \mathrm{~kg} / \mathrm{m}^{2}$ ) and $40-90 \%$ have been shown to be overweight $\left(\mathrm{BMI}>25 \mathrm{~kg} / \mathrm{m}^{2}\right)[3]$.

\section{Hypothalamus/pituitary-ovarian axis dysfunction}

A large proportion of PCOS women have high LH levels and normal or decreased levels of FSH, resulting in an increased LH/FSH ratio. The prevalence of an increased LH/ FSH ratio is partly related to BMI and it is more prevalent in PCOS women of normal weight and less common with increasing BMI [3]. Partly due to the increased LH stimulation there is increased ovarian androgen production mainly from the ovarian theca cells (PCO women have thicker hypertrophic theca cells in their ovarian follicles compared to others) $[1,3,4]$. The follicular steroid secretion follows the two-cell cooperation or theory where LH-stimulated theca cells produce mainly androstenedione from the steroid precursor cholesterol and via pregnenolone, progesterone and 17-OH-progesterone. The high androgen levels were suggested as the main cause of the ovarian follicle maturation arrest with subsequent appearance of the characteristic multiple small ovarian follicles with $\geq 12$ follicles measuring 2-9 $\mathrm{mm}$ in diameter during ultrasound scanning of PCO. Figure 3 shows ultrasound or sonographic appearance of PCO with $\geq 12$ follicles measuring $2-9 \mathrm{~mm}$ in diameter.

\section{Sex hormone binding globulin (SHBG)}

Sex hormone binding globulin is produced by the liver, and PCOS women have decreased levels of SHBG which is caused by the inhibitory effects of insulin on SHBG production. In addition, overweight/obesity decreases SHBG production more with subsequent increased levels of free (non-binding) biologically active androgens [3].

\section{Insulin resistance, dyslipidemia and metabolic syndrome}

Insulin resistance means a reduced glucose response to insulin with a subsequent increase in insulin secretion (hyperinsulinemia) and utilization of fat as a source of en- ergy through lipolysis, which leads to elevated triglyceride and decreased HDL cholesterol [1]. Insulin resistance is a prominent feature of PCOS and is associated with elevated triglycerides, decreased HDL cholesterol, IGT, and CVDs $[1,16]$. Insulin resistance in PCOS women was first noted by Burghen et al. and several subsequent studies confirmed the close association between disturbed insulin metabolism, IR and PCOS [17]. In addition, Burghen et al. found a significant positive correlation between insulin, testosterone and androstenedione levels among PCOS women. Insulin stimulates ovarian androgen secretion, and maintains ovarian hyperandrogenism in PCOS through the direct effect of insulin on ovarian steroidogenesis or due to its effect on luteinizing hormone (LH) receptors of the theca cells [18].

This explains the association between hyperinsulinemia and the severity of hirsutism suggested by Landay et al. in PCOS women [19]. Excess androgen interferes with the maturation of the ovarian follicles with subsequent arrest of the follicular growth and anovulation. Sikka et al. found a significant positive correlation between IR, hyperinsulinemia in PCOS and both the ovarian size and the number of follicles per ovary [18]. The glucose/insulin ratio is suggested as a useful screening test for IR in PCOS women [16].

\section{Endometrial and breast cancers}

Endometrial, ovarian and breast cancer are mainly discussed as having a possible association with PCOS. Obesity, hyperinsulinemia, null parity and long exposure to unopposed estrogen are common features of PCOS and are risk factors for endometrial cancers. The mechanism behind endometrial cancer in PCOS is the unopposed stimulation of the endometrium by estrogens, which can cause endometrial hyperplasia with increased risk of atypia and eventually endometrial cancer [3]. A recent meta-analysis showed an almost three times higher risk of developing endometrial cancer for PCOS women (odds ratio $=2.70$; 95\% CI: 1.00-7.29) compared to non-PCOS women [3].

In addition, obesity, hyperandrogenism, long unopposed estrogen exposure and a long period of infertility are common features of PCOS and are risk factors for breast cancers, but most of the studies of PCOS and breast cancer show no increased risk of breast cancer for women with PCOS [1, 3].

\section{Hyperandrogenism}

Clinical hyperandrogenism of PCOS is diagnosed by acne and/or hirsutism, which means the presence masculine hair pattern distribution (upper lip, chin, chest, upper abdomen and chest) [3]. Mostafa et al. found that the most common manifestation of hyperandrogenism in PCOS is hirsutism, which is reported in up to $70 \%$ of PCOS women [11]. Figure 4 shows masculine hair pattern distribution (upper lip, chin) in a woman with PCOS and Figure 5 shows a woman with PCOS presenting with acne.

\section{Insulin resistance and obesity}

Obesity and/or acanthosis nigricans (dark hyperpigmented area on the back of the neck, under the breast or on inner thighs) is a characteristic sign for IR and spillover 


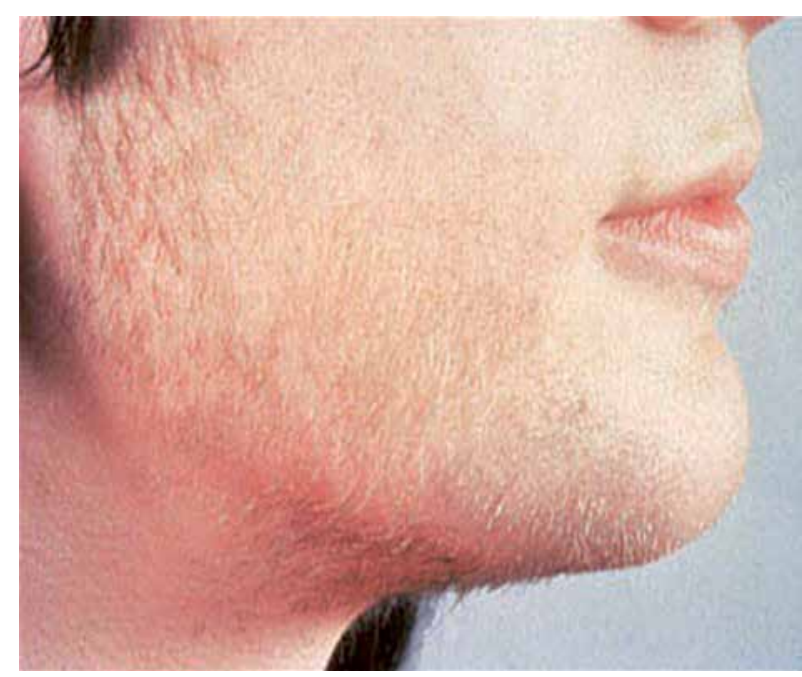

Figure 4. Masculine hair pattern distribution (upper lip, chin) in PCOS woman

of the high insulin to the skin (not a characteristic sign for PCOS). At least $35 \%$ of women with PCOS are obese and the obesity of PCOS women is characterized by BMI $>26 \mathrm{~kg} /$ $\mathrm{m}^{2}$ with increased waist circumference $>35$ inches. In obese PCOS women the fat is deposited in the upper body areas such as the arms, waist and abdominal region in contrast to normal feminine fat which is normally deposited in the lower body areas such as the hips, thighs and buttocks.

\section{Dyslipidemia, CVDs and metabolic syndrome}

Elevated triglyceride and decreased HDL cholesterol are common findings in PCOS due to insulin resistance and utilization of fat as a source of energy through lipolysis [1, 19]. The National Cholesterol Education Program Adult Treatment Panel (NCEP ATP III) guidelines defined the metabolic disturbance as having at least three of the following abnormalities: increased waist circumference, high blood pressure, elevated fasting blood glucose, low serum high-density lipoprotein (HDL) cholesterol and hyperglycemia $[18,19]$.

Two hundred and ninety-five (295) premenopausal Chinese PCOS women diagnosed according to the Rotterdam criteria and 98 controls without PCOS were studied by Cheung et al. for the prevalence of metabolic syndrome (MS) and CVDs and they found that the MS was more prevalent in overweight and obese (41.3\%) than normal weight PCOS women $(0.9 \%)$ [20].

Similarly, Hahn et al. found that the prevalence of the MS and CVDs was 33.8\% in German PCOS women and they found that the prevalence of MS in German PCOS women increased with obesity and age [21].

A systematic review conducted by Moran et al. concluded that hyperandrogenic anovulatory PCOS is associated with more adverse metabolic profile including greater total and abdominal obesity, insulin resistance and risk factors for CVDs [22].

Recently; Pikee et al. concluded that all phenotypes of PCOS had a deranged endocrine and metabolic profile compared to their controls but the prevalence of IR and MS was maximum in hyperandrogenic phenotypes [23].

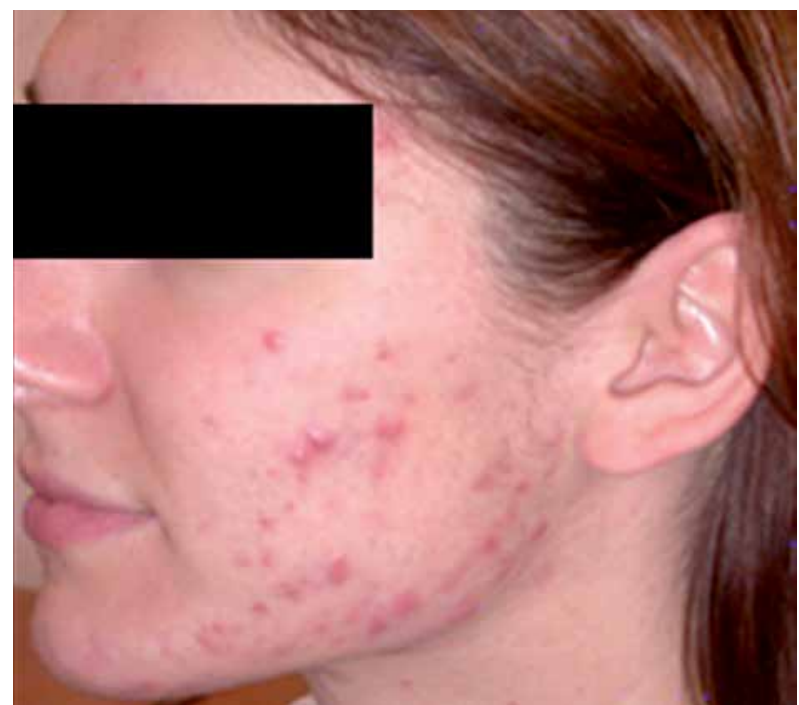

Figure 5. PCOS woman presenting with acne

\section{Reproductive consequences}

\section{Infertility}

Three quarters of PCOS women are sub-fertile or infertile, which is mostly due to oligo/anovulation and hyperandrogenism. The high androgen levels were suggested as the main cause behind the ovarian follicle maturation arrest with subsequent anovulation and infertility $[11,16,24]$.

\section{Dysfunctional uterine bleeding (DUB)}

It is mainly due to long exposure to unopposed estrogen secretion from the multiple small ovarian follicles, which predispose to endometrial hyperplasia with atypia and subsequent endometrial cancers.

A systematic review conducted by Goodman et al. concluded that women with PCOS have multiple risk factors with pregnancy including glucose intolerance, gestational diabetes, gestational hypertension, preeclampsia and premature births. Goodman et al. also concluded that women with PCOS should be screened and treated for hypertension and diabetes prior to conception [24].

\section{Polycystic ovary syndrome and ovarian reserve}

Ovarian reserve describes the woman's reproductive ability, through identification of the number and quality of oocytes available in her ovaries [25]. Ovulation induction (OI) and/or in-vitro-fertilization (IVF) are commonly used for infertility treatment in PCOS women. Ovarian reserve assessment helps to detect the PCOS women's ovarian response to exogenous gonadotropins stimulation during ovulation induction and/or IVF treatment. Decreased ovarian reserve (DOR) refers to women whose ovarian response to exogenous gonadotropin stimulation is reduced compared to similar women of the same age [25].

Methods of evaluation of the ovarian reserve include:

1) Cycle-day 3 serum follicle stimulating hormone (FSH) and estradiol $\left(\mathrm{E}_{2}\right)$ : cycle-day 2-4 FSH commonly used to measure the ovarian reserve. $\mathrm{FSH}>10-20 \mathrm{mIU} / \mathrm{ml}$ 
associated with DOR [25]. Serum $\mathrm{E}_{2}$ is not used alone for assessment of the ovarian reserve and its measurement should be combined with measurement of the serum FSH [25]. Normal serum FSH with elevated $\mathrm{E}_{2}$ $>60 \mathrm{pg} / \mathrm{ml}$ in follicular phase associated with DOR and increased rate of IVF cycle cancellation.

2) Clomiphene citrate challenge (CCC) test: the CCC test involves assessment of the FSH on day 3 and day 10 of the menstrual cycle after $100 \mathrm{mg}$ clomiphene citrate (CC) daily from day 5 to day 9 of the menstrual cycle. High serum FSH after clomiphene citrate stimulation suggests DOR [25].

3) Antral follicle count (AFC): AFC assessment by trans-vaginal ultrasound (TVS) done in early follicular phase of the menstrual cycle. The AFC assessment involves detection of the follicles measuring 2-10 $\mathrm{mm}$ in diameter in the ovaries. Mean AFC of $5.2 \pm 2.1 \mathrm{SD}$ (standard deviation) is associated with DOR [25]. In addition, the AFC is increased in PCOS women and decreased in women receiving combined contraceptive pills [25].

4) Serum anti-Mullerian hormone (AMH): $\mathrm{AMH}$ is secreted by follicular granulosa cells of the antral follicles, is gonadotropin-independent and therefore can be measured on any day of the cycle [25].

The AMH decreases with external use of contraceptive pills or gonadotrophic releasing hormones ( $\mathrm{GnRH}$ ) and hypogonadotropic hypogonadism [25]. The AMH $<1 \mathrm{ng} / \mathrm{ml}$ is usually associated with DOR and poor IVF outcome. The AMH is increased in PCOS women [25]. The PCOS women have higher ovarian reserve compared to others of the same age and PCOS women are liable to ovarian hyperstimulation syndrome (OHSS) during the controlled ovarian hyper-stimulation $(\mathrm{COH})$ protocols of the IVF cycles $[3,25]$.

\section{Psychological consequences of polycystic ovary syndrome}

Polycystic ovary syndrome affects quality of life and can worsen the existing anxiety and depression either due to the features of PCOS or due to the diagnosis of a chronic disease. Previous studies concluded that the prevalence of depression symptoms and mood disorders is high in most obese PCOS women.

In addition, previous studies concluded that emotional distress and depression were significantly higher in PCOS women with high BMI and waist-to-hip ratio (WHR) compared to their controls [24, 25].

Polycystic ovary syndrome women have clinical and/ or biochemical signs of hyperandrogenism and several studies have shown a positive correlation between depression and hirsutism. It has been suggested that PCOS women have lower self-esteem, a more negative self-image and have higher levels of depression and psychological distress owing to the physical appearance characteristics of hyperandrogenism (hirsutism and acne) and obesity $[24,25]$.

\section{Treatment of polycystic ovary syndrome}

There is no ideal therapy that treats all the clinical features of PCOS, so the treatment in PCOS is directed towards the clinical presentation (targeted therapy):

1) Effective weight reduction by $5-10 \%$ in PCOS women is associated with significant improvement and regression of the reproductive features of PCOS [1].

2) Pharmacological therapy in PCOS includes: 1) oral contraceptive pills (OCP) which regulate the menstrual cycle, improve hyperandrogenism through increased production of the hepatic SHBG with subsequent decrease of the free androgen and decrease the ovarian androgen production through suppression of LH secretion [3]. 2) Metformin (insulin sensitizer) reduces insulin resistance in PCOS women; metformin side effects include gastrointestinal symptoms and it is contraindicated in women with serum creatinine $\geq 1.4 \mathrm{mg} / \mathrm{dl}$ and liver diseases $[4,25]$.

Metabolic consequences

1) Effective weight loss by $5-10 \%$.

2) Metformin (insulin sensitizers).

Reproductive consequences

1) PCOS women presenting with oligomenorrhoea and amenorrhea are treated as follows:

- Effective weight loss (5-10\%) and metformin.

- OCP or cyclic progesterone for 2-3 months.

2) PCOS women presenting with infertility are treated as follows:

- PCOS women should reduce their weight prior to conception.

- Ovulation induction (OI) using clomiphene citrate and/ or gonadotrophins.

- OI with insemination (IUI)

- In-vitro fertilization/intracytoplasmic sperm injection (IVF/ICSI).

Psychological consequences

The psychological consequences in PCOS women are mainly due to obesity, acne and hirsutism

1) Reassurance with psychological support.

2) Effective weight loss by $5-10 \%$.

3) Acne treated by:

- Cosmetic therapy.

- Medical treatment if the cosmetic therapy is ineffective in the form of OCP or OCP with anti-androgen including cyproterone acetate for 6 months.

4) Hirsutism treated by:

- Cosmetic therapy and laser therapy.

- Medical treatment if the cosmetic therapy is ineffective in the form of OCP or OCP with anti-androgen including cyproterone acetate for 6 months.

\section{Conclusions}

Polycystic ovary syndrome is the most common endocrine disorder affecting women in their reproductive 
age and different phenotypes of PCOS exist, based on the PCOS definition by NIH and ESHRE/ARM and the clinical presentation. The increased ovarian androgen production in PCOS women due to the increased LH stimulation were suggested as the main cause of the ovarian follicle maturation arrest with subsequent infertility and characteristic appearance of the PCO during an ultrasound scan. Significant positive correlations between insulin, testosterone and androstenedione levels are observed among PCOS women, which explains the association between hyperinsulinemia and the severity of hirsutism in PCOS women. Effective weight reduction by $5-10 \%$ in PCOS women is associated with significant improvement of the reproductive features of the syndrome.

\section{Conflict of interest}

The authors declare no conflict of interest.

\section{References}

1. Abdelazim IA, Farok Elsawah W. Metabolic syndrome among infertile women with polycystic ovary syndrome. Asian Pac J Reprod 2015; 4: 44-8.

2. Dumesic DA, Abbott DH, Padmanabhan V. Polycystic ovary syndrome and its developmental origins. Rev Endocr Metab Disord 2007; 8: 127-41.

3. Schmidt J, Landin-Wilhelmsen K, Brännström M, Dahlgren E. Cardiovascular disease and risk factors in PCOS women of postmenopausal age: a 21-year controlled follow-up study. J Clin Endocrinol Metab 2011; 96: 3794-803.

4. Rotterdam ESHRE/ASRM-Sponsored PCOS Consensus Workshop Group. Revised 2003 consensus on diagnostic criteria and longterm health risks related to polycystic ovary syndrome (PCOS). Hum Reprod 2004; 19: 41-7.

5. Xita N, Georgiou I, Lazaros L, Psofaki V, Kolios G, Tsatsoulis A. The synergistic effect of sex hormone-binding globulin and aromatase genes on polycystic ovary syndrome phenotype. Eur J Endocrinol 2008; 158: 861-5.

6. Xita N, Georgiou I, Lazaros L, Psofaki V, Kolios G, Tsatsoulis A. The role of sex hormone-binding globulin and androgen receptor gene variants in the development of polycystic ovary syndrome. Hum Reprod 2008; 23: 693-8.

7. Yang F, Ruan YC, Yang YJ, et al. Follicular hyperandrogenism downregulates aromatase in luteinized granulosa cells in polycystic ovary syndrome women. Reproduction 2015; 150: 289-96.

8. Zhang CW, Zhang XL, Xia YJ, et al. Association between polymorphisms of the CYP11A1 gene and polycystic ovary syndrome in Chinese women. Mol Biol Rep 2012; 39: 8379-85.

9. Jin JL, Sun J, Ge HJ, et al. Association between CYP19 gene SNP rs2414096 polymorphism and polycystic ovary syndrome in Chinese women. BMC Med Genet 2009; 10: 139.

10. Sun J, Fan H, Che Y, et al. Association between ACE gene I/D polymorphisms and hyperandrogenism in women with polycystic ovary syndrome (PCOS) and controls. BMC Med Genet 2009; 10: 64.

11. Mostafa RA, Al-Sherbeeny MM, Abdelazim IA, et al. Relation between aromatase gene CYP19 variation and hyperandrogenism in polycystic ovary syndrome Egyptian women. J Infert Reprod Biol 2016; 4: 1-5
12. Chen J, Shen S, Tan Y, et al. The correlation of aromatase activity and obesity in women with or without polycystic ovary syndrome. J Ovarian Res 2015; 8: 11.

13. Wang $\mathrm{H}, \mathrm{Li} \mathrm{Q}$, Wang $\mathrm{T}$, et al. A common polymorphism in the human aromatase gene alters the risk for polycystic ovary syndrome and modifies aromatase activity in vitro. Mol Hum Reprod 2011; 17: 386-91.

14. Zhang XL, Zhang CW, Xu P, et al. SNP rs2470152 in CYP19 is correlated to aromatase activity in Chinese polycystic ovary syndrome patients. Mol Med Rep 2012; 5: 245-9.

15. Medeiros SF, Barbosa JS, Yamamoto MM. Comparison of steroidogenic pathways among normoandrogenic and hyperandrogenic polycystic ovary syndrome patients and normal cycling women. J Obstet Gynaecol Res 2015; 41: 254-63.

16. Mostafa R, Al-Sherbeeny MM, Abdelazim IA, Elshehawy Y, Wahba KA, Abuel-Fadle A. Frequency of insulin resistance in Egyptian women with polycystic ovary syndrome. MOJ Womens Health 2015; 1: 32-6.

17. Burghen GA, Givens JR, Kitabrhi AE. Correlation of hyperandiogenism with hyperinsulinism in polycystic ovarian disease. J Clin Endocrinol Metab 1980; 50: 113-6.

18. Sikka P, Gainder S, Dhaliwal LK, Bagga R, Sialy R, Sahdev S. Ultrasonography of the ovaries and its correlation with clinical and endocrine parameters in infertile women with PCOS. Int J Fertil Womens Med 2007; 52: 41-7.

19. Landay M, Huang A, Azziz R. Degree of hyperinsulinemia, independent of androgen levels, is an important determinant of the severity of hirsutism in PCOS. Fertil Steril 2009; 92: 643-7.

20. Cheung LP, Ma RC, Lam PM, et al. Cardiovascular risks and metabolic syndrome in Hong Kong Chinese women with polycystic ovary syndrome. Hum Reprod 2008; 23: 1431-8.

21. Hahn S, Tan S, Sack S, et al. Prevalence of the metabolic syndrome in German women with polycystic ovary syndrome. Exp Clin Endocrinol Diabetes 2007; 115: 130-5.

22. Moran L, Teede H. Metabolic features of the reproductive phenotypes of polycystic ovary syndrome. Hum Reprod Update 2009; 15: 477-88.

23. Pikee S, Shivani S, Jayshree B. Endocrine and metabolic profile of different phenotypes of polycystic ovarian syndrome. J Obstet Gynaecol India 2016; 66 (Suppl 1): 560-6.

24. Goodman NF, Cobin RH, Futterweit W, Glueck JS, Legro RS, Carmina E; American Association of Clinical Endocrinologists (AACE); American College of Endocrinology (ACE); Androgen Excess and PCOS Society. American Association of Clinical Endocrinologists, American College of Endocrinology, and Androgen Excess and PCOS Society Disease State Clinical Review. Guide to the best practices in the evaluation and treatment of polycystic ovary syndrome - part 2. Endocr Pract 2015; 21: 1415-26.

25. Practice Committee of American Society for Reproductive Medicine. Diagnostic evaluation of the infertile female: a committee opinion. Fertil Steril 2012; 98: 302-7. 